$R M x A C, \mathbf{5 3}, 108-112(2021)$

(c) 2021: Instituto de Astronomía, Universidad Nacional Autónoma de México

https://doi.org/10.22201/ia.14052059p.2021.53.22

\title{
THE GRB PROMPT OPTICAL EMISSION FEATURES ON THE EXAMPLE OF GRB160625B
}

\author{
V. V. Topolev ${ }^{1}$ and V. M. Lipunov ${ }^{1}$
}

\section{RESUMEN}

Este trabajo presenta un modelo de observaciones cuasiperiódicas que pudieran observarse en contrapartidas ópticas de los estallidos de rayos gamma (GRB). Este modelo está basado en la hipótesis de que el GRB ocurra en un sistema binario con un viento estelar intenso como ingrediente adicional. El progenitor sería una estrella de helio que emite un viento estelar intenso perturbado por el objeto compacto (estrella de neutrones). Se han calculado los parámetros del modelo usando GRB160625B como ejemplo, para el que la red global MASTER obtuvo una curva de luz con alta resolución temporal.

\section{ABSTRACT}

This article discusses a model for the occurrence of quasiperiodic oscillations in the optical light curve of a gamma-ray burst(GRB). The model is based on the assumption that GRB occurs in a binary system with strong stellar wind. Progenitor is a small core of helium star which emits a strong stellar wind that perturbs by the compact companion (neutron star).There is a calculation of model parameters by using the GRB160625B as an example, for which the MASTER global network received an optical curve with high time resolution.

Key Words: gamma ray burst: individual: GRB160625B — stars: jets — stars: wind, outflows

\section{INTRODUCTION}

\subsection{Detection and observation}

On June 25, 2016 at 22:40:16 of UT, the Fermi Gamma-ray Space Telescope(GBM) detected a burst of gamma radiation, which was a soft precursor of GRB. After 31 seconds, the Russian robot-telescope of the Global Network MASTER, Lomonosov Moscow State University, located in the Canaries ${ }^{2}$ received a message from Fermi and after another 26 seconds,started observing the error box with optical cameras.

Despite the large errors in the primary coordinates of the GRB, the entire error box fell into the MASTER VWF - twin VERY Wide Field Cameras with 800 square degrees FOV up to $14^{m}$, groundbased analogue of VWF cameras installed on board the Lomonosov space observatory and is specifically designed for such situations. Therefore, MASTER was already "looking" at the right place when, 131 seconds after the first report, the NASA space observatory detected very bright main burst. Another node of the global network Master joined the observation - Crimean MASTER-Tauris ${ }^{3}$. 12 seconds

\footnotetext{
${ }^{1}$ Moscow State University, Moscow, Universitetsky pr. 13, Moskow 119992, Russia.

${ }^{2}$ Observatory of the Institute of Astrophysics of the Canary Islands, Tenerife, Spain.

${ }^{3}$ Lomonosov MSU Crimean Astronomical station(SAI), Russia.
}

after receiving the refined coordinates at 22:44:30 MASTER-Tauris obtained the first frames.

Within the sample of nearly 2,000 bursts detected by the GBM, only six other events have a comparable duration. Also this GRB is one of the most bright GRB's in visible band ever. Incredible duration of this event and the fact that MASTER VWF has a high time resolution(the exposure time is 5 seconds) allowed us to get a unique curve in visiblel band. Moreover for the first time in the history of the study of gamma-ray bursts, the polarization of the optical radiation of the gamma-ray burst was registered at the moment when the flash was still continuing. Measurements of polarization showed that the prompt phase is produced via fast-cooling synchrotron radiation in a large-scale magnetic field that is advected from the black hole and distorted by dissipation processes within the jet (Fig.1(Troja et al. 2017)).

\subsection{Hypothesis}

In this paper, we consider one of the hypotheses that could explain quasiperiodic oscillations of the light curve of GRBs, which occurs in a binary system. A possible confirmation of the existence of such oscillation is the light curve of GRB160625b. Even taking into account the fact that the presence of quasi-oscillations is not reliable in the case of GRB160625b, this hypothesis is interesting for 


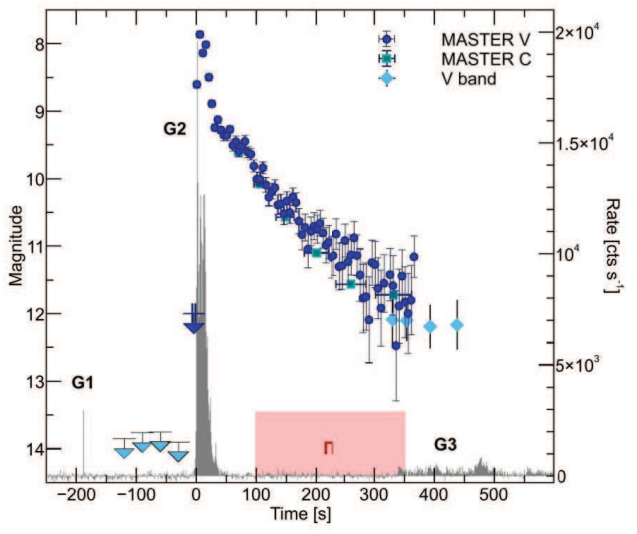

Fig. 1. The background-subtracted GBM light curve (black, 10-250 keV) consists of three main episodes: a short, spectrally soft precursor (G1), a bright and spectrally hard main burst (G2), and a fainter and longer lasting tail of emission (G3), Optical data from the MASTER Net telescopes. The red box marks the time interval over which polarimetric measurements were carried out(Troja et al. 2017).

consideration because a number of theoretical works lead to the conclusion that gamma-ray bursts should occur in binary systems(Tutukov \& Cherepashchuk 2016)(Tutukov 2018). The hypothesis is based on the assumption that colapsed star has strong stellar wind perturbed by the companion star. The jet passes through an inhomogeneous medium and produces flashes in high-density zones. These flashes are superimposed on the overall trend of GRBs, which is relatively smooth.

\section{FIRST MODEL(A)}

\subsection{Observational data}

To study the structure of the stellar wind, it is necessary to go to the reference frame associated with the collapsing star. Taking into account the scale factor(redshift of GRB160625b - $z=1.406$ ), the time intervals are recalculated according to the equation (1). $d t_{0}$ is observed time interval; $d t_{\text {rest }}$ is time interval in rest reference frame.

$$
\frac{d t_{\text {rest }}}{d t_{0}}=\frac{1}{1+z}
$$

We assume that the energy intensity of the flash is directly proportional to the density and energy pumped by the jet. In this way it is convenient to convert stellar magnitudes into relative energy units $\left(\frac{E}{E_{\max }}\right)$ by taking the maximum radiation flux equal to one. As you can see in the new plot (Fig. 2) energetic curve in blue rectangle has significant deviation

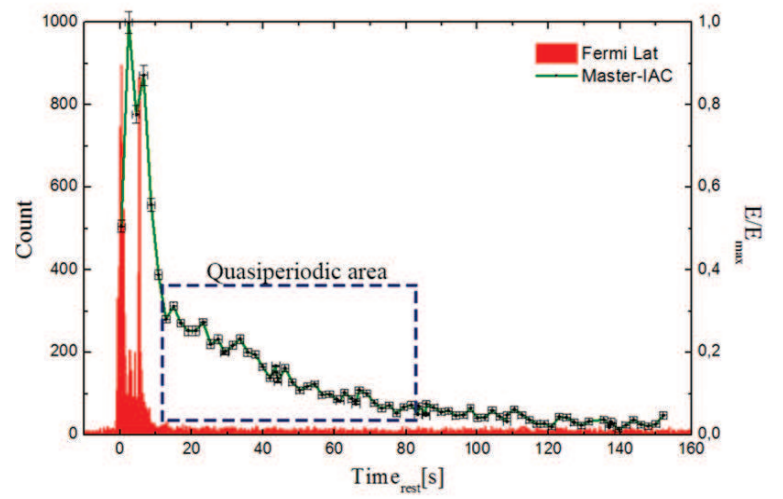

Fig. 2. Green showing relative energetic curve of GRB160625B in the rest reference frame, taking into account the scale factor. (calculated from MASTER observations). Red bars - Fermi LAT( $>10 \mathrm{MeV})$.

from the trend with a relatively small error. We can select some "peaks", the period of which ones has similar values. This may indicate that they are not random.

\subsection{Scenario}

To model the process and calculate the parameters of a binary star system, we need to determine the perturbation mechanism, which create asymmetric density distribution of stellar wind. Consider one of the simplest mechanisms - gravitational interaction of the companion star directly onto stellar wind particles(Collisionless celestial mechanics). This mechanism severely limits the set of possible scenarios. We have two basic requirement:

- Collapsing star must emit a strong stellar wind.

- The system must has small semi-major axis(the effect is inversely proportional to the square of the distance)

After investigating several scenarios we chose a system consisting of a dense and compact helium star and a neutron star, because helium stars mostly have strong stellar wind and neutron star can provide a small size of the semi-major axis.

\subsection{Limitation on the system}

Mass Scenario(set of masses that were used for modeling): $M_{N s} \in[1.3-1.5] M_{\odot}$ - the mass range of neutron star; $M_{\mathrm{He}} \in[5-15] M_{\odot-}$ the mass range of helium star. The minimum size of the semi-major axis is limited by the filling of the helium star of its Roche lobe. In mathematical terms, this condition is shown by the equation (2). $r_{\text {Roche }}$ - Roche lobe radius; $R$ - distance between helium star and neutron 
star (eccentricity $=0 \Rightarrow R$ - semi-major axis); $R_{0}$ radius of helium star. $r_{\text {Roche }} \geq R_{0}$.

$$
r_{\text {Roche }}=R\left(1-\left(\frac{\alpha}{3}\right)^{\frac{1}{3}}\right), \quad \alpha=\frac{M_{N s}}{M_{H e}+M_{N s}}
$$

Using the semi-major axis and masses, we can calculate the period of system. Besides using mass-radius relation, we can exclude one parameter. Example of such a theoretical relationship - (Woosley 2019).

\subsection{Velocity law}

Also we must set the law of acceleration of stellar wind. Observational studies widely use the beta velocity law(eq. 3) and usually consider $\beta$ as a free parameter; $R_{0}$ - the radius of a star; $v_{\infty}$ is the speed of stellar wind at infinity. This is the most common model, and there are more detailed and complex improvements (Krtička \& Kubát 2011).

$$
v=v_{\infty}\left(1-\frac{R_{0}}{r}\right)^{\beta}
$$

The functions $v(r)$ can be associated with the force $F(r)$. Simple example:

$$
\beta=\frac{1}{2}, \quad A=\text { const }: \quad F=\frac{A}{r^{2}}
$$

$\beta$ can vary widely. Some examples of approximations: $\beta=0.7-1$ for O stars (Puls et al. 1996). For some stars (for example, early B supergiants), $\beta$ can reach values $\beta=3$ (Crowther et al. 2006).

\subsection{Modeling and results}

The simulations were performed using Python. The simulations used $10^{7}$ test particles of the stellar wind. Variable parameters:

$$
\begin{aligned}
& \text { - } M_{H e} \\
& \text { - } M_{N s} \\
& \text { - } R_{0} \\
& \text { - } v_{\infty}
\end{aligned}
$$

The result of the calculations is a diagram of the distribution of the density of stellar wind particles, which depends on the above parameters - For example Fig. 3 and Fig.4. The size of the bin has the same value as the time resolution of observations (speed of jet $\approx \mathrm{c}$ ).

Assuming that the jet has a significant projection on the plane of the orbit, consider the density curve on the ray of view and different position angles - For example Fig.5, Fig.6 and Fig.7.

After superimposing the density distribution on the overall trend, we will have model light curve. One of the best simulating light curve in Fig.8. Period of flash has same value, but very big deviation. Calculated parameters:

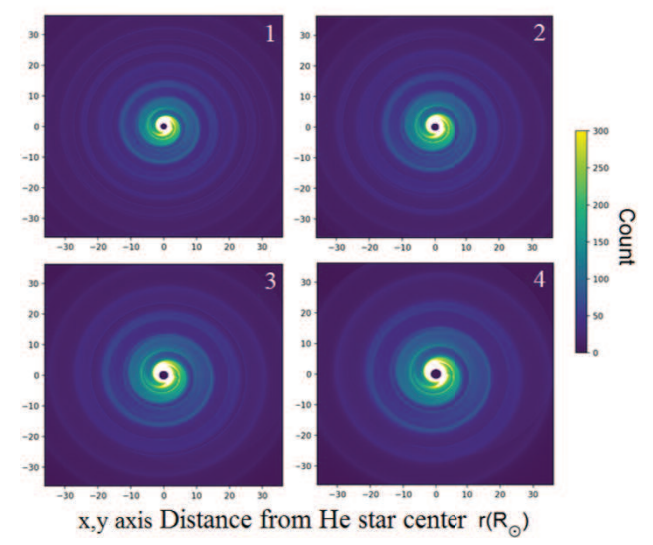

Fig. 3. Distribution of particle density as a function of star radius. $1-1 R_{\odot} ; 2-1.2 R_{\odot} ; 3-1.4 R_{\odot} ; 4-1.6 R_{\odot}$ $\left(R_{0}\right)$

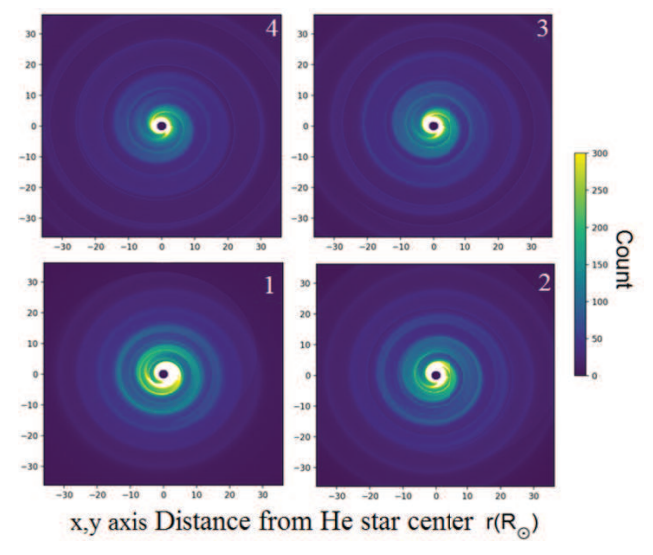

Fig. 4. Distribution of particle density as a function of wind speed at infinity. $1-1000 \mathrm{~km} \mathrm{~s}^{-1}, 2-1200 \mathrm{~km} \mathrm{~s}^{-1}$, $3-1400 \mathrm{~km} \mathrm{~s}^{-1}, 4-1600 \mathrm{~km} \mathrm{~s}^{-1}\left(v_{\infty}\right)$

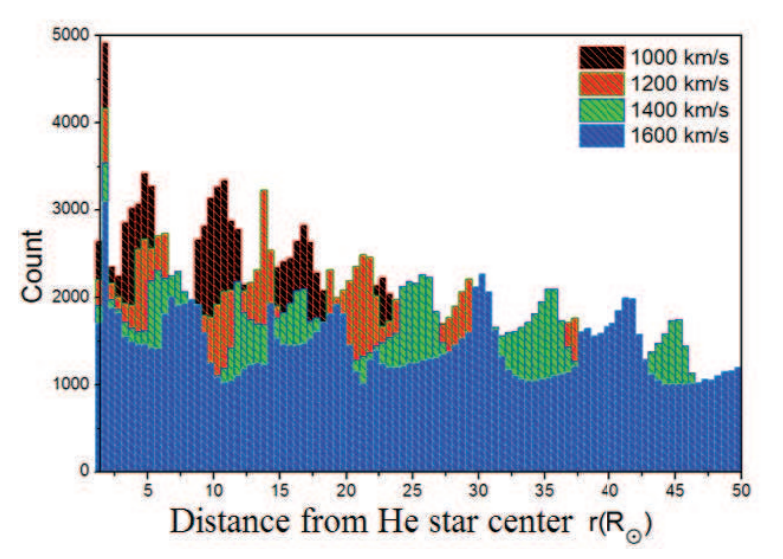

Fig. 5. Density histograms for different $v_{\infty}$ in a narrow beam. Size of binning $\approx 0.9 R_{\odot}$. 


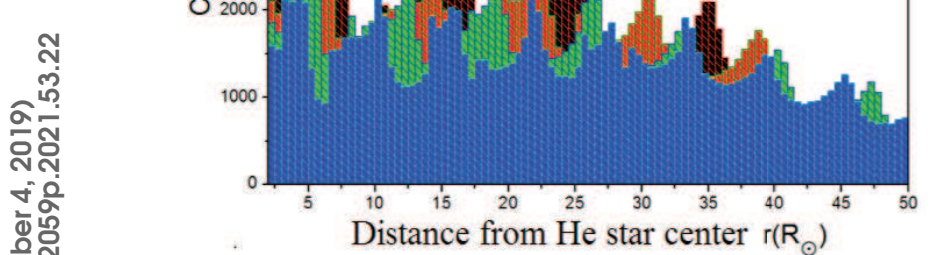

Fig. 6. Density histograms for different $R_{0}$ in a narrow beam. Size of binning $\approx 0.9 R_{\odot}$.

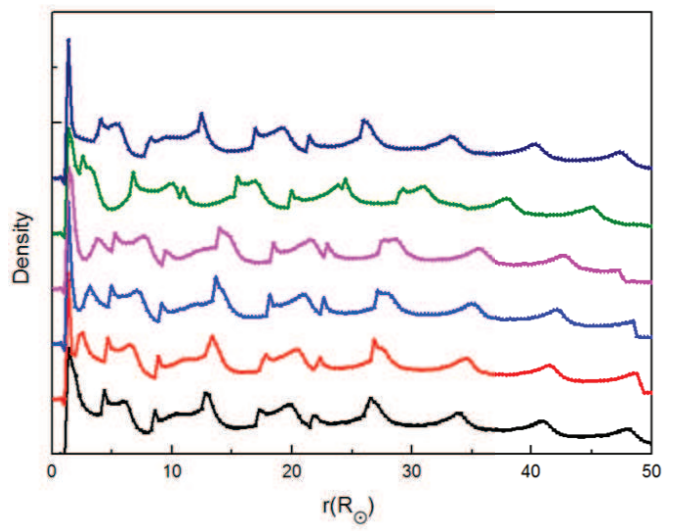

Fig. 7. Density distribution for the same set of parameters, but for different directions of jet $\left(\operatorname{step}=\frac{\pi}{3}\right)$

- $M_{N s} \approx 1.3 M_{\odot}$

- $M_{H e} \approx 15 M_{\odot}$

- $R_{0} \approx 1.2 R_{\odot}$

- $v_{\infty} \approx 1240 \mathrm{~km} \mathrm{~s}^{-1}$

- $\beta=\frac{1}{2}$

\subsection{Problems of the first model}

As you can see our model has a number of significant drawbacks:

1. Direction of Jet - most likely the direction of the jet coincide with the axis of rotation, perpendicular to the plane of the orbit $\Rightarrow$ projection is minimal

2. Collisionless celestial mechanics gives quite sharp pattern, which should be smoothed taking into account the interaction of wind particles with each other.

3. Low confidence in the comparison of model and observed curves.

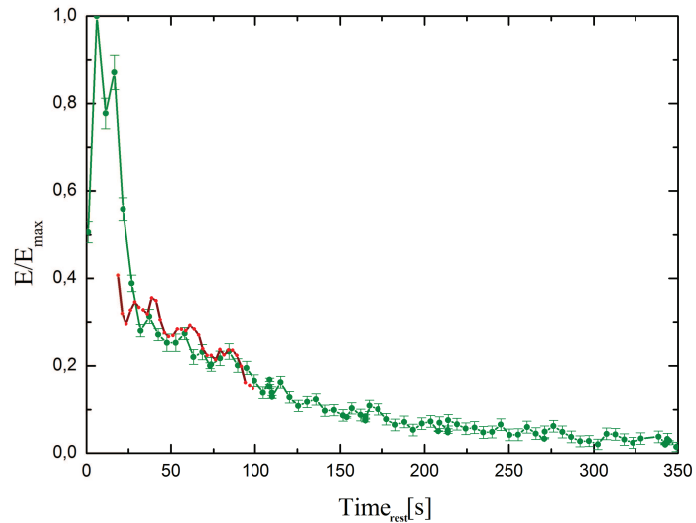

Fig. 8. Model A light curve(Red) in comparison with the observed(Green).

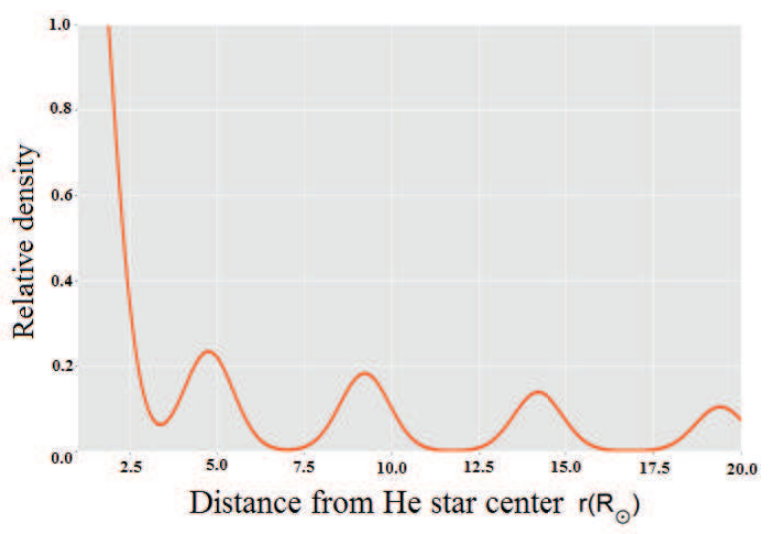

Fig. 9. Propagation of density waves in the system. Red curve describes the relative density depending on the distance to the center of the helium star.

Conclusion is that we need a new model.

\section{SECOND MODEL(B)}

A companion star (NS) orbits in an ellipse around the collapsing star, passing through the periapsis of the orbit, and perturbing the main star. The density of the stellar wind stream increases, creating a density wave that is already accelerating according to the law of stellar wind acceleration, which was described above. In this case wave of density can spread in any direction. This model does not provide a detailed description of the stellar wind perturbation process, but use simple assumptions.

\subsection{Results of model $B$}

The main parameters of this model are $\beta, v_{\infty}$, $T_{\text {sys }}$. The masses of the components and $\mathrm{R}$ in this approximation determinated period of system, there can be a lot of variation. We also assume that at 


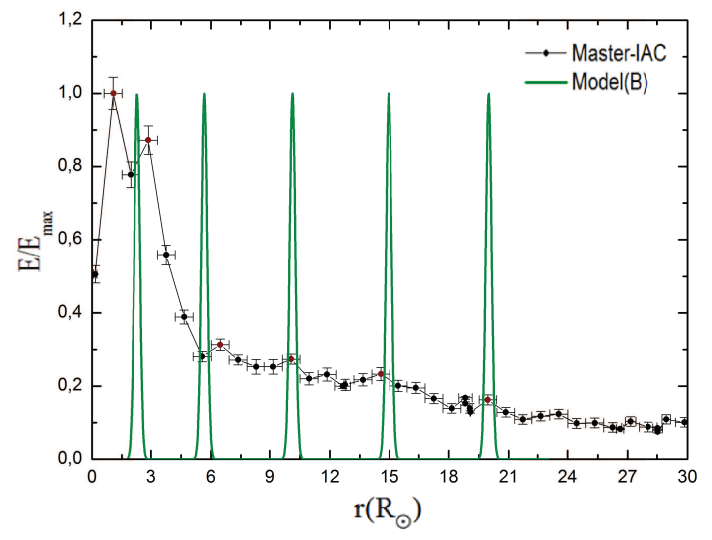

Fig. 10. Overlay of the model B peaks (green) on the observations (black).

every moment of time the density wave has a Gauss form. Minimizing deviations gives Fig.10 and set of parameters:

- $M_{N s} \approx 1.5 M_{\odot}$

- $M_{H e} \approx 15 M_{\odot}$

- $R_{0} \approx 1.4 R_{\odot}$

- $v_{\infty} \approx 1320 \mathrm{~km} \mathrm{~s}^{-1}$

- $T_{\text {sys }}=1.6 \mathrm{~h}$

- $\beta=1.4$

\section{CONCLUSION}

We have considered two models $\mathrm{A}$ and $\mathrm{B}$, and the first one has a number of significant drawbacks. Model B, without a precise description of the mech- anism of creation an asymmetric pattern of stellar wind distribution, can describe features of the light curve and has quite probable parameters of the binary system. The existence of a quasiperiodic of the light curve may indicate the duality of the system where the GRB occurred. In some of the models of stellar evolution the duality of the system almost is a necessary condition for the appearance of GRBs (Tutukov \& Cherepashchuk 2016), (Tutukov 2018).

\section{REFERENCES}

Castor, J. I., Abbott, D. C., \& Klein, R. I. 1975, ApJ, 195,157

Chandrasekhar, S. 1934, MNRAS, 94, 522

Crowther, P. A., Lennon, D. J., \& Walborn, N. R. 2006, A\&A, 446, 279

Gorbovskoy, E. S., Lipunov, V. M ., Buckley, D. A. H., et al. 2016, MNRAS, 455, 3312

Krtička, J., \& Kubát, J. 2011, A\&A, 534, A97

Milne, E. A. 1926, MNRAS, 86, 459

Puls, J., Kudritzki, R.-P., Herrero, A., et al. 1996, A\&A, 305,171

Rublev, S. V. 1965, AZh, 42, 718

Troja, E., Lipunov, V. M., Mundell, C. G., et al. 2017, Natur, 547, 425

Tutukov, A. V., \& Cherepashchuk, A. M. 2016, ARep, 60, 461

Tutukov, A. V. 2018, A. A. Boyarchuk Memorial Conference, 143

Woosley, S. E. 2019, ApJ, 878, 49 